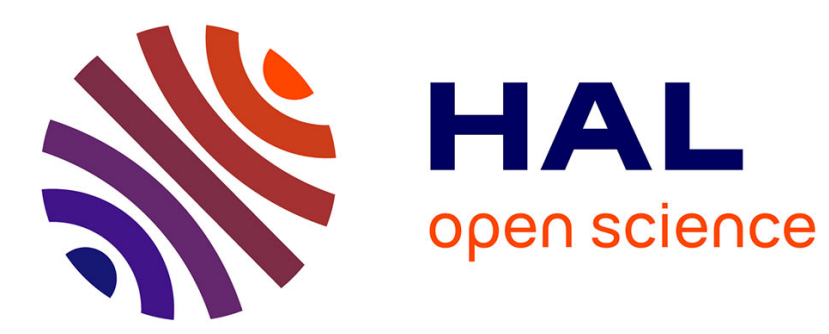

\title{
Computational Brain Connectivity Mapping: A Core Health and Scientific Challenge
}

Rachid Deriche

\section{To cite this version:}

Rachid Deriche. Computational Brain Connectivity Mapping: A Core Health and Scientific Challenge. Medical Image Analysis, 2016, 10.1016/j.media.2016.06.003 . hal-01335669

\section{HAL Id: hal-01335669 \\ https://hal.science/hal-01335669}

Submitted on 22 Jun 2016

HAL is a multi-disciplinary open access archive for the deposit and dissemination of scientific research documents, whether they are published or not. The documents may come from teaching and research institutions in France or abroad, or from public or private research centers.
L'archive ouverte pluridisciplinaire HAL, est destinée au dépôt et à la diffusion de documents scientifiques de niveau recherche, publiés ou non, émanant des établissements d'enseignement et de recherche français ou étrangers, des laboratoires publics ou privés. 


\title{
Computational Brain Connectivity Mapping: A Core Health and Scientific Challenge
}

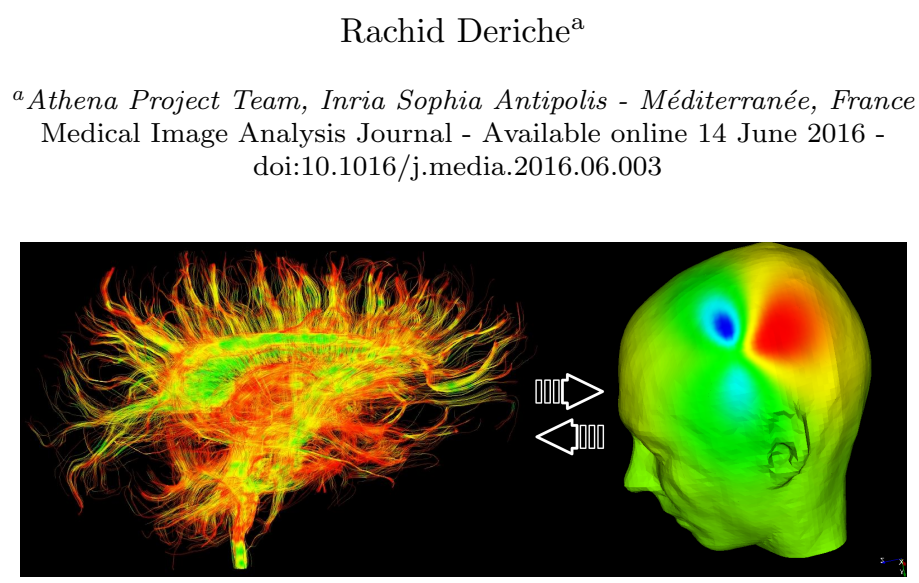

\begin{abstract}
One third of the burden of all the diseases in Europe is due to problems caused by diseases affecting brain. Although exceptional progress have been obtained for exploring the brain during the past decades, it is still terra-incognita and calls for specific efforts in research to better understand its architecture and functioning. To take up this great challenge of modern science and to solve the limited view of the brain provided just by one imaging modality, this article advocates the idea developed in my research group of a global approach involving new generation of models for brain connectivity mapping and strong interactions between structural and functional connectivities. Capitalizing on the strengths of integrated and complementary non invasive imaging modalities such as diffusion Magnetic Resonance Imaging (dMRI) and Electro \& MagnetoEncephalography (EEG \& MEG) will contribute to achieve new frontiers for identifying and characterizing structural and functional brain connectivities and to provide a detailed mapping of the brain connectivity, both in space and time. Thus leading to an added clinical value for high impact diseases with new perspectives in computational neuro-imaging and cognitive neuroscience.
\end{abstract}

Keywords: Diffusion MRI, MEG, EEG, Structural and Functional Brain Connectivity, Brain Mapping, Networks.

Email address: Rachid.Deriche@inria.fr (Rachid Deriche) 


\section{Introduction}

In its reports published in (Andlin-Sobocki et al., 2005, Wittchen et al. 2011), the European Brain Council (EBC) investigated the socio-economic impact of brain diseases on European society and concluded that about one third of the burden of all diseases in Europe is due to brain diseases. Every year, over a third of the total EU population suffers from mental disorders and the burden of brain diseases (neurological, neurosurgical and psychiatric diseases together) in Europe is estimated to cost $€ 798$ billion per year. Both EBC reports project that this burden will further increase in the coming decades due to the ageing of the European society and conclude that for now and in the near future there is a very strong societal and economical need for improvement in diagnosis and therapy of brain diseases. They also call for increased efforts in basic and clinical research and strongly advocate the need for new models and computational approaches in neuroscience and neuroimaging to better understand the brain architecture and functioning.

\section{Mapping the human brain : a core health ambition of modern science}

Mapping the brain architecture and functioning is a core health ambition of the 21st century and one of the greatest challenges of modern science. The human brain is one of the most impressive and complex structures in the known universe. With approximately 85 to 100 billion neurons, the human brain is definitely an object of prodigious complexity. However, more important than the number of neurons is the extraordinary complex brain circuitry formed by the 1.000 trillion possible synaptic connections between the neurons. Since the work of pioneers such as Wernicke and Dejerine, and following Geschwind's revolutionary disconnectionist framework, many important neurological diseases and disorders including Alzheimer's, Schizophrenia, Autism, Brain trauma, Epilepsy and others have been shown to be related to pathological alterations in the connectivity of the brain (Johansen-Berg and Behrens, 2009).

This is why ambitious worldwide scientific efforts to understand the hierarchical, complex, architectural and functional network organization of the brain, are combined to conduct huge data generation projects in the USA (Human Connectome Project ${ }^{1}$ and Brain Research through Advancing Innovative Neurotechnologies (BRAIN) Initiative ${ }^{2}$ and models integration project in Europe (FET Flagship Human Brain Project 3 Slightly smaller but equally ambitious complementary initiatives have been launched around the globe such as China's Brain Database to identify clues to tackling brain diseases and disorders, Japan's Brain/MINDS (Brain Mapping by Integrated Neurotechnologies

1 http://www.humanconnectomeproject.org/

2 http://www.nih.gov/science/brain/

3 https://www.humanbrainproject.eu 
for Disease Studies) project, Israel Brain Technologies, Brain Canada, AusBrain (Australia) to name just a few. These large initiatives and brain research projects differ in scope and detail but all have been developed to federate efforts to improve our understanding of the brain.

Due to the vast amount of complex data to acquire, broad range of scales at which they have to be considered, huge variety of problems to tackle and models to integrate, projects as HCP, HBP, BRAIN etc are only a first step and there is clearly a long way to go to achieve the aims of precisely understanding the structure and functioning of the brain. Although exceptional progress has been obtained for exploring the brain during the past decades, the contribution from the exact sciences in this endeavor is still relatively less than what it should be, not to say insufficient and more appropriate models and robust methods for identifying and characterizing structural and functional brain connectivity are still lacking while very much needed. By providing the right mathematical and computational tools as well as the rigorous methodologies, exact sciences may indeed offer substantial added value for exploring the brain, still terra incognita and last continent remaining to discover. In addition, although various new insights from individual imaging modalities have been obtained for brain diseases, it is very clear that an effective research approach is to use various noninvasive imaging modalities and capitalize on the strengths of each of them to solve the limited view of the brain provided separately by any imaging modality.

\section{BCM : Brain Connectivity Mapping}

During the last decade, huge progress has been made with non-invasive and in vivo medical imaging technologies, such as diffusion and functional magnetic resonance image (dMRI, fMRI) (Buxton, 2002) as well as electro and magneto-encephalography (EEG \& MEG jointly denoted as M/EEG) to reconstruct the hierarchical complex structural and functional network organization of the brain. Multi-modal imaging, integrating functional (fMRI \& M/EEG) with structural (dMRI) descriptions has the potential to yield a detailed picture of brain architecture and dynamics and dramatically improve our understanding of brain connectivities. A broad range of studies have shown links between anatomical and functional whole-brain connectivity. However, there has been limited work on a systematic framework to investigate whole-brain interactions between structure and function.

A large amount of work has already been devoted to joint analysis methods for dMRI and fMRI data, Hence, a significant review study of dMRI and fMRI data fusion methodologies and their applications in cognitive and clinical neurosciences can be found in Zhu et al. (2014). The large amount of relevant literature published on dMRI and fMRI has been estimated to include hundreds of papers (at the date of March 2013) with methods categorized into three classes: fMRI assists dMRI, dMRI assists fMRI, and joint dMRI and fMRI fusion. This interest in combining dMRI and fMRI will certainly continue to grow and I envision that effective multi-modalities fusion will play increasingly 
important roles in neuroimaging and brain sciences in the years to come. See for instance Castellanos et al. (2013) where clinical applications of the functional connectome are considered with a focus on how resting-state fMRI methods can lead to biomarker identification for brain disorders and to Griffa et al. (2013) where interesting highlights and outlooks of structural connectomics for clinical applications in brain diseases are discussed.

However, if a large amount of work has been devoted to joint analysis methods for dMRI and fMRI data, much less work, not to say none, has been devoted for dMRI and M/EEG. Therefore, to advance our current fragmentary and limited understanding of the brain, and solve the limited view of the brain provided just by one imaging modality, I push forward the idea to map the brain connectivity with new generation of models and well appropriate noninvasive neuro-imaging modalities such as diffusion Magnetic Resonance Imaging (dMRI) \& Electro and Magneto-Encephalography (EEG \& MEG jointly as M/EEG) methods. At Inria, my research group ATHENA actively contributes in the field of non-invasive and multimodal brain imaging and has acquired a remarkable joint expertise in the modeling and analysis of dMRI \& M/EEG data, which is clearly fundamental to non-invasively examine connections through combinations of functional and anatomical imaging techniques.

During the last decade, my research group has pushed far forward the stateof-the-art in both dMRI \& M/EEG, developing new computational models and tools, tackling and solving a set of important and challenging problems to unleash the full power and multivariate information content of dMRI \& M/EEG data. Combining the measurements obtained by these two types imaging modalities has the potential of providing a detailed view both in space and time of the functioning brain at a macroscopic level. Capitalizing on the strengths of dMRI $\&$ M/EEG and building on the bio-physical and mathematical foundations of our models will bring a remarkable and significant added clinical value to identify and characterize brain connectivity and positively impact brain diseases. In that spirit, I summarize in the sequel some challenges, with a particular emphasis on those related to dMRI, and target areas for the upcoming years and the rationale behind them.

\section{Advanced dMRI for structural connectivity}

To better understand the brain, it is important first to focus on its structural connectivity and to rely on diffusion MRI (dMRI) which is the unique in vivo and non-invasive technique capable of providing the structural connectivity information and investigating the complex microstructure of the cerebral white matter. Introduced in the mid 80 's, dMRI is well adapted to detect, characterize and quantify possible white matter abnormalities of brain tissues that cannot be revealed by standard imaging techniques (Jones, 2011; Johansen-Berg and Behrens, 2009).

To take up the challenge to precisely and accurately recover and characterize the structural brain connectivity and map it with ever-increasing detail and 
richness, it is important to develop generative and ground-breaking models for the advanced acquisition, processing and analysis of dMRI data. Overall, these last twenty years have seen an explosion of intensive scientific research which has vastly improved and literally changed the face of dMRI. These ground breaking changes range from improved and more powerful gradients, scanner-technology to acquisition, post-processing and reconstruction schemes, resulting in shorter acquisitions and more accurate modeling of the brain-tissues microstructure and connectivity. However, although great improvements have been made in the last twenty years (Assaf et al., 2008, Zhang et al., 2012, Descoteaux et al. 2011, Ozarslan et al. 2013), major improvements are still required primarily to optimally acquire dMRI data, better understand the biophysics of the signal formation, recover invariant and intrinsic microstructure features, identify biophysically important bio-markers and improve tractography. For short, there is still considerable room for improvement to take dMRI from the benchside to the bedside.

Some recents results and debates in the dMRI community perfectly illustrate this importance and the vital need to develop ground-breaking models for recovering a precise and accurate cerebral connectivity. Indeed, recently, Van Wedeen $\&$ al. (Wedeen et al., 2012) have analyzed relationships of adjacency and crossing between cerebral fiber pathways in four nonhuman primate species and in humans by using dMRI. Their results suggest that the cerebral fiber pathways adhere to a simple and well organized three-dimensional geometric structure consisting of sheets of fibers that intersect at right angles like Manhattan-like grids. However, this point of view is not totally shared within the community, as pointed out by Catani \& al. (Catani et al., 2012) who argue that Wedeen's method is likely to miss fibers crossing at angles less than about 70 degrees. They claim also that this view is clearly biased by the limits of their techniques, and conclude that our brain circuitry is wired more like London's chaotic tangle than Manhattan's organized grid. These results and debates clearly illustrate the challenge and complexity of recovering a precise and accurate cerebral connectivity, and the importance and the vital need to develop accurate and precise computational models and tools to help correctly answer such kind of concerns.

Today, it is well understood that more accurate models and better signal descriptions are necessary to overcome the limitations of these state-of-the-art signal reconstruction and tractography methods. Due to the ambiguities of low dMRI spatial resolution, complexities of the underlying tissue and uncertainties of signal noise, newer and more accurate dMRI models have to be found and developed. In my research group, we have recently contributed to design optimal acquisition schemes for single and multiple q-shell in diffusion MRI (Deriche et al., 2009, Caruyer et al., 2013). In particular, it is our optimal sampling scheme (Caruyer et al., 2013) freely available for download 4 (Caruyer et al. 2013), which has been used in the HCP (Human Connectome Project). We have also exploited the ability of Compressed Sensing to recover the whole 3D dMRI

${ }^{4}$ http://www.emmanuelcaruyer.com/q-space-sampling.php 
signal and some of its important features from a limited number of samples (Merlet and Deriche, 2013). However, and up to now, in all our acquisitions schemes as well in those developed and used in the community, the gradient directions are optimized by considering only geometrical constraints i.e without taking into account any possible a-priori information related to the data and/or to the scanner. We believe it is important to revisite the important problem of the acquisition and tackle the challenging issue to develop more appropriate and efficient sampling schemes, in particular in some specific situations where we could have some prior knowledge on data and/or the used scanner.

The dMRI signal is highly complex, hence, the mathematical tools required for processing it have to be commensurate in their complexity. The dMRI signal is often analyzed partially (single q-shell or QBI) or more globally (DSI or multi-q-shell) and in various representations such as spherical coordinates or Cartesian coordinates. Hence, it is important to explore a number of new and sophisticated mathematical approaches to improve the quality, accuracy and efficiency of modeling the dMRI signal (Ghosh and Deriche, 2016). Historically, the first model in dMRI is the 2nd order diffusion tensor (DTI) (Basser et al., 1994) which assumes the EAP to be Gaussian centered at the origin. While practical, this assumption limited the ability of the DTI to describe complex, singular and intricate fiber configurations (U-shape, kissing or crossing fibers). To overcome this limitation, so-called Diffusion Spectrum Imaging (DSI) and High Angular Resolution Diffusion Imaging (HARDI) methods such as Q-ball imaging and other multi-tensors, compartment models and high order tensor models were developed to resolve the orientationnality of more complicated fiber bundle configurations (Schultz et al., 2013; Jones, 2011, Johansen-Berg and Behrens, 2009: Ghosh et al. 2013: Ghosh and Deriche, 2009).

In particular, the exploration of high order tensors and the development of the theory of tensor invariants as a mathematical framework for computing new bio-markers for dMRI offer exciting and innovative research direction to better characterize white matter abnormalities (Ghosh and Deriche, 2009, Schultz et al., 2013). High order tensors will also allow to go beyond simple bio-markers, such as those derived from the classical second order diffusion tensor. Indeed, as of today, DTI based bio-markers such as Mean Diffusivity (MD) and Fractional anisotropy (FA) are those clinically used at this date but are well known to be definitely sensitive to confounding factors such as partial volume and axonal dispersion, severely lack specificity and do not capture the subtle effects that might be early indicators of diseases. New biomakers from high order tensors will provide new insights to better understand the structural connectivity and help characterize important neurodegenerative diseases (Papadopoulo et al. 2014, Caruyer and Verma, 2015).

High order dMRI modeling allow also to infer and use micro-structural features, such as axonal diameter and density. Indeed, dMRI which is capable to measure the displacement of water molecules at the scale of tens of microns, is exquisitely sensitive to any change in tissue micro-structure and can provide unique insights and intrinsic parameters to precisely characterize white matter, in particular abnormalities that underpin diseases states. Tissue micro-structure 
parameters extracted from high order dMRI models could be used as important a-priori information in a unified and efficient micro-structure based tractography algorithm. This clearly pave the way to a new generation of tractography algorithms and opens new perspectives for better reconstruct anatomical brain connectivity and impact on certain neurodegenerative diseases thanks to a better connectivity quantification. (Sherbondy et al., 2010; Girard et al., 2015).

All these challenging scientific problems, when solved, will lead to direct measurements of important micro-structural based biomakers from high order model providing much greater and new insights to better understand the structural connectivity and help characterize important neurodegenerative disease mechanisms, recovery and development.

\section{Advanced M/EEG for Functional Connectivity}

A second important challenge is to complement brain structural connectivity with brain functional connectivity. Indeed, if a better knowledge of the structural wiring of the brain is essential to move ahead, it is clearly not sufficient to understand the functioning of the brain. For instance, if one consider the tiny worm C. elegans, one of the simplest organisms with a nervous system, we are far from fully understanding its brain even though, the wiring diagram of its 302 only neurons has been comprehensively mapped with a connectome shown to be a small-world network.

dMRI provides only structural connectivity but, as of today, gives no clue on time-varying functional connectivity. Indeed, existing dMRI methods for mapping structural connectivity have provided numerous valuable insights but still remain inadequate to provide information about directionality. On the other hand, MEG and EEG (jointly denoted as M/EEG) provide (after source reconstruction) time courses of the activity of the cortical areas from which it is possible to compute dynamic connectivity measures. Indeed, M/EEG are two non-invasive neurophysiological techniques which directly record electrical voltages from electrodes placed on the scalp (EEG) and magnetic field from SQUID sensors placed above the head (MEG). Both are fundamentally related through Maxwell's equations to the distribution of dipole moment throughout the brain and head and hence have similar millisecond temporal resolution and are very important functional neuroimaging modalities for studying the dynamics of neural activities

MEG and EEG can be measured simultaneously and reveal complementary properties of the electromagnetic fields of the brain (Hämäläinen et al., 1993) for studying the dynamics of neural activities. Contrarily to fMRI, which "only" measures an haemodynamic response linked to the metabolic demand, MEG and EEG measure a direct consequence of the electrical activity of the brain.

While the few hundred time signals obtained using M/EEG have a clear clinical interest, the measurements are made outside, or on the surface of the head, and hence only provide partial information on the localization of the sources of activity. With a proper model of the head and the sources of this 
electromagnetic activity, it is possible to simulate the electrical propagation (forward problem (Kybic et al., 2005, Gramfort et al., 2010) ) and to recover the sources corresponding to measurements using an inverse problem. Solving the inverse problem is the key to identify and localize brain areas of the observed activity (Baillet et al. 2001) and developing ground-breaking tools and advanced $\mathrm{M} / \mathrm{EEG}$ source reconstruction is one of the major objectives to achieve to push far forward the state-of-the-art in M/EEG.

\section{Toward sructural and functional brain connectivity mapping}

dMRI provides a global and systematic view of the long-range structural connectivity within the whole brain. On the opposite, M/EEG measures brain activation over time and provides, after source reconstruction (solving the socalled inverse problem of source reconstruction), time courses of the activity of the cortical areas. Given its very high temporal resolution, the signal of reconstructed sources can be processed to reveal the functional connectivity between the nodes.

Such measures are however limited by source estimation methods that currently do not leverage any information on structural connectivity. Such information could improve functional connectivity by disambiguating multiple solutions corresponding to different hypotheses on how a given brain network operates. While dMRI and M/EEG have been the object of considerable research separately, there have been very few studies on combining the information they provide. Some existing studies deal with the localization of abnormal MEG signals, particularly in the case of epilepsy, and on studying the white matter fibers near the detected abnormal source but to our knowledge there are very few studies merging data coming both from M/EEG and dMRI at the analysis level (Philippe et al., 2012, Belaoucha et al., 2014; Philippe et al., 2014).

Combining the structural and functional information provided by dMRI and $\mathrm{M} / \mathrm{EEG}$ is a difficult problem as the spatial and temporal scales of the two types of measures are extremely different. Nonetheless, combining the measurements obtained by these two techniques can potentially provide a detailed macroscopic view of the functioning brain both in space and time. All these considerations support the need to develop new models and computational tools for dMRI and M/EEG and integrate their information to reconstruct brain connectivities.

To complement the above challenges who's components are mainly concerned with developing sophisticated techniques for acquiring, processing and analyzing complex and multidimensional dMRI \& M/EEG data, the different theoretical approaches to be developed above will have to be more closely integrated into a unified computational framework. This could be performed through the construction of a joint dynamical structural-functional network of the brain that will help to identify and characterize brain connectivity and see how far the developed framework can be get close to explaining high-level brain structure and function. Advanced network analysis and machine learning tools will have to be developed to design theoretically well founded methods and metrics for de- 
veloping, analyzing and validating this joint and dynamical anatomo-functional network,

This challenge will lead to the creation of a robust and complete connectivity characterization of patients and controls. The joint structural-functional network developed will define a unique relationship between structure and function thanks to the directionality information and the edgewise quantification of connectivity grounded on fine spatial domain resolution, micro-structure features and high order tensors for dMRI and on exquisite time domain sensitivity and high order models for M/EEG.

Last but not least, the advanced integrated and post-hoc analysis tools for the joint structural-functional connectivity analysis are expected to help elucidate patterns of abnormality in the studied population of patients by providing a measure of abnormal connectivity that can then be correlated with clinical measures of symptom severity, aiding in prognosis.

\section{Conclusion}

In this article, I described some of the challenges recently solved and identified some of those still to be solved to achieve new frontiers in brain connectivity mapping through dMRI and M/EEG. Even if the described vision includes objectives for the upcoming years that can be considered as extremely challenging and very ambitious 5 . I am confident that the use of structural and functional brain connectomics to identify outcome biomarkers for brain disorders will greatly extend the capabilities of neuroimaging in cognitive and clinical neurosciences and that important progress with significant scientific results and clinical impact will occur in support of these objectives. This will push forward and far beyond the state-of-the-art measurements and modeling approaches that span across the disciplines of computer sciences, applied mathematics, neuroimaging and health science.

\section{References}

Andlin-Sobocki, P., Jönsson, B., Hans-Ulrich Wittchen, H., and Olesen, J. (2005). Costs of disorders of the brain in europe. European Journal of Neurology, 12(1).

Assaf, Y., Blumenfeld-Katzir, T., Yovel, Y., and Basser, P. J. (2008). Axcaliber: a method for measuring axon diameter distribution from diffusion mri. Magnetic Resonance in Medicine, 59(6):1347-54.

Baillet, S., Mosher, J., and Leahy, R. (2001). Electromagnetic brain mapping. IEEE Signal Processing Magazine, 18(6):14-30.

5 The work leading to the objectives listed in this article has received funding from the European Research Council (ERC) under the European Union's Horizon 2020 research and innovation program (ERC Advanced Grant agreement No 694665 to start on Sept. 1st, 2016.) 
Basser, P. J., Mattiello, J., and Le Bihan, D. (1994). Mr diffusion tensor spectroscopy and imaging. Biophysical Journal, 66(1):259-267.

Belaoucha, B., Lina, J.-M., Clerc, M., Philippe, A.-C., Grova, C., and Papadopoulo, T. (2014). Using diffusion mri information in the maximum entropy on mean framework to solve meg/eeg inverse problem. In Proceedings of Biomag, Halifax, Canada.

Buxton, R. B. (2002). Introduction to Functional Magnetic Resonance Imaging. Cambridge University Press.

Caruyer, E., Lenglet, C., Sapiro, G., and Deriche, R. (2013). Design of multishell sampling schemes with uniform coverage in diffusion mri. Magnetic Resonance in Medicine, 69(6):1534-1540.

Caruyer, E. and Verma, R. (2015). On facilitating the use of $\{$ HARDI $\}$ in population studies by creating rotation-invariant markers. Medical Image Analysis, 20(1):87 - 96 .

Castellanos, F. X., Martino, A. D., Craddock, R. C., Mehta, A. D., and Milham, M. P. (2013). Clinical applications of the functional connectome. Neuroimage., 80:527-540.

Catani, M., Bodi, I., and F., D. (2012). Comment on "the geometric structure of the brain fiber pathways". Science, 337(6102):1605.

Deriche, R., Calder, J., and Descoteaux, M. (2009). Optimal real-time q-ball imaging using regularized kalman filtering with incremental orientation sets. Medical Image Analysis, 13(4):564-579.

Descoteaux, M., Deriche, R., Le Bihan, D., Mangin, J.-F., and Poupon, C. (2011). Multiple q-shell diffusion propagator imaging. Medical Image Analysis, 15(4):603-621.

Ghosh, A. and Deriche, R. (2009). From second to higher order tensors in diffusion-mri. In Aja-Fernández, S., de Luis García, R., Tao, D., and Li, X., editors, Tensors in Image Processing and Computer Vision, Advances in Pattern Recognition, chapter 9, pages 315-. Springer London.

Ghosh, A. and Deriche, R. (2016). A survey of current trends in diffusion mri for structural brain connectivity. Journal of Neural Engineering, 13(1).

Ghosh, A., Milne, T., and Deriche, R. (2013). Constrained diffusion kurtosis imaging using ternary quartics \& mle. Magnetic Resonance in Medicine. Article first published online: 2 JUL 2013 - Volume 71, Issue 4, April 2014, Pages: 1581-1591.

Girard, G., Fick, R., Descoteaux, M., Deriche, R., and Wassermann, D. (2015). Axtract: Microstructure-driven tractography based on the ensemble average propagator. In The 24th biennial international conference on Information 
Processing in Medical Imaging (IPMI), Springer Lecture Notes Computer Science (LNCS) series., pages 675-686.

Gramfort, A., Papadopoulo, T., Olivi, E., and Clerc, M. (2010). OpenMEEG: opensource software for quasistatic bioelectromagnetics. BioMedical Engineering OnLine, 9(45).

Griffa, A., Baumann, P. S., Thiran, J.-P., and Hagmann, P. (2013). Structural connectomics in brain diseases. NeuroImage, 80:515-526.

Hämäläinen, M., Hari, R., IImoniemi, R. J., Knuutila, J., and Lounasmaa, O. V. (1993). Magnetoencephalography - theory, instrumentation, and applications to noninvasive studies of the working human brain. Reviews of Modern Physics, 65(2):413-497.

Johansen-Berg, H. and Behrens, T. E., editors (2009). Diffusion MRI : From Quantitative Measurement to In vivo Neuroanatomy. Elevier - Academic Press.

Jones, D. K., editor (2011). Diffusion MRI: Theory, Methods, and Applications. Oxford University Press.

Kybic, J., Clerc, M., Abboud, T., Faugeras, O., Keriven, R., and Papadopoulo, T. (2005). A common formalism for the integral formulations of the forward eeg problem. IEEE Transactions on Medical Imaging, 24:12-28.

Merlet, S. and Deriche, R. (2013). Continuous diffusion signal, eap and odf estimation via compressive sensing in diffusion mri. Medical Image Analysis, $17(5): 556-572$.

Ozarslan, E., Koay, C., Shepherd, T., Komlosh, M., Irfanoglu, M., Pierpaoli, C., and Basser, P. J. (2013). Mean apparent propagator (MAP) MRI: a novel diffusion imaging method for mapping tissue microstructure. Neuroimage, $78: 16-32$.

Papadopoulo, T., Ghosh, A., and Deriche, R. (2014). Complete set of invariants of a 4th order tensor: the 12 tasks of hardi from ternary quartics. In Medical Image Computing and Computer-Assisted Intervention - MICCAI, volume 17, pages 233-240, Boston, USA.

Philippe, A.-C., Clerc, M., Papadopoulo, T., and Deriche, R. (2012). A nested cortex parcellation combining analysis of meg forward problem and diffusion mri tractography. In IEEE International Symposium on Biomedical Imaging (ISBI), pages 518-521, Barcelona. IEEE.

Philippe, A.-C., Papadopoulo, T., Bénar, C., Badier, J.-M., Clerc, M., and Deriche, R. (2014). Propagation of epileptic spikes revealed by diffusion-based constrained meg source reconstruction. In 19th International Conference on Biomagnetism (BIOMAG 2014), Halifax, Canada. 
Schultz, T., Fuster, A., Ghosh, A., Deriche, R., Florack, L., and Lim, L.-H. (2013). Higher-order tensors in diffusion imaging. In Westin, C.-F. and Burgeth, B., editors, Visualization and Processing of Tensors and Higher Order Descriptors for Multi-Valued Data. Springer. Dagstuhl Reports.

Sherbondy, A., Rowe, M., and Alexander, D. (2010). Microtrack: an algorithm for concurrent projectome and microstructure estimation. In et al., T. J., editor, Med Image Comput Comput Assist Interv. (MICCAI), Part I. LNCS 6361, pages 183-190. Springer-Verlag.

Wedeen, V. J., Rosene, D. L., Wang, R., Dai, G., Mortazavi, F., Hagmann, P., Kaas, J. H., and Tseng, W.-Y. (2012). The geometric structure of the brain fiber pathways. Science, 335(6076):1628-1634. See also the corrected version : Corrections and Clarifications, Science 11 May 2012: Vol. 336, Issue 6082, pp. 670 , DOI: $10.1126 /$ science.336.6082.670.

Wittchen, H., Jacobi, F., Rehm, J., Gustavsson, A., Svensson, M., Jönsson, B., Olesen, J., Allgulander, C., Alonso, J., Faravelli, C., Fratiglioni, L., Jennum, P., Lieb, R., Maercker, A., van Os, J., Preisig, M., Salvador-Carulla, L., Simon, R., and Steinhausen, H.-C. (2011). Ecnp/ebc report 2011 - the size and burden of mental disorders and other disorders of the brain in europe 2010. European Neuropsychopharmacology, 21:655-679.

Zhang, H., Schneider, T., Wheeler-Kingshott, C. A., and Alexander, D. C. (2012). NODDI: practical in vivo neurite orientation dispersion and density imaging of the human brain. NeuroImage, 61:1000-1016.

Zhu, D., Zhang, T., Jiang, X., Hu, X., Chen, H., Yang, N., Lv, J., Han, J., Guo, L., and Liu, T. (2014). Fusing dti and fmri data: A survey of methods and applications. NeuroImage, 102(Part 1):184-191. 